\title{
COMPARATIVE STUDY: SCHOOL TRIP AND SCIENTIFIC EDUCATIONAL ACADEMIC TRIP IN THE NORTH-WEST OF ROMANIA
}

\author{
BIANCA SORINA RĂCĂŞAN \\ „Babeş-Bolyai" University, Faculty of Geography, Cluj-Napoca, Romania, \\ e-mail: bianca_racasan@yahoo.com \\ VALERIU MIRCEA VANA \\ „Babeş-Bolyai" University, Faculty of Geography, Cluj-Napoca, Romania, \\ e-mail: valeriu_mircea@yahoo.com
}

(Received: January 2015; in revised form: July 2015)

\begin{abstract}
The current study proposes a comparative approach of two forms of non-formal educational activities: the school trip and the scientific educational academic trip with their similarities and differences regarding both tourist and pedagogical levels. In order to acquire this goal the following objectives have been set: analysing a geographicaltourist itinerary, analysing the involved tourist services, analysing the trip planner for the specified itinerary, analysing certain teaching activities, differentiated based on the level of study (pre-university/superior), analysing students and undergraduates' worksheets after they have been completed according to the requirements. Consequently a series of similarities have appeared between the school trip and the academic trip, especially related to the theme, purpose, evaluation form, participants' involvement degree, structure and integrated tourist elements (from attractions to services). On the other hand, the main differences that were highlighted, starting with educational level, age difference, and participants' number have had repercussions on the quantitative and qualitative particularities of the tasks and last but not least, on the evaluation product (students' portfolios and undergraduates' trip notebooks).
\end{abstract}

Keywords: pre-university education, superior/academic education, non-formal educational activities, educational tourism, trip planner, worksheets, portfolios, academic trip notebooks 


\section{INTRODUCTION}

Setting up an itinerary either for the roadway or for a specialised thematic tourist route involves anticipating some aspects, the more numerous as the complexity level gets higher. Thus, making the project for a geographic school or academic trip, over a period of several days, will always imply more effort and preparation on behalf the organisers than a simple visit made by a family or a group of friends to a single tourist sight. In this context, each element of the school or academic trip will be selected according to students/undergraduates' interest, travel purpose, geographic region and the existing tourist attractions.

All throughout the school years, as students, we have all attended at least one school trip. Further on, as undergraduates of Faculty of Geography (Tourism Geography Specialisation), we took part in field trips, developing the skills to conceive a tourist product in the form of a tour, starting from an ordinary geographic itinerary. Noticing the existence of similarities and differences between a school trip and an academic one, emerged both the need and interest to compare these two non-formal activities - from the point of view of the organising party - unfolded within identical circumstances derived from the same itinerary.

The objectives of the study are: the analysis of a geographic-tourist itinerary, the analysis of the provided tourist services, the analysis of the trip planner, the analysis of the teaching activities differentiated by the level of education (pre-university/university); the analysis of certain worksheets, the comparison between some forms of non-formal education: the school trip and the academic trip.

\section{THEORETICAL SUBSTANTIATION}

The Romanian educational system is organised and functions in compliance with the provisions of the Law of National Education (2011). The final purpose of children's and young people's education is to form skills, considered "multifunctional a transferable ensembles of knowledge, abilities and skills" (idem, p. 2). The formation and development of specific and general abilities is achieved through a polyvalent instructive-educational process conducted on several levels and in various forms. Blându (2008), Bocoş and Jucan (2008a) emphasize the fact that education is characterised by a threefold structure which can express itself in either a formal, non-formal or informal manner.

Cozma (1988, apud Bradea, 2012, p. 145) considers that the meaning of the term non-formal represents "a less formalised or non-formalised educational reality, which always has formative effects". The purpose of nonformal education is to respond to the educational needs of a group, having 
nonetheless clear learning objectives (Costea, 2009, p. 10). The particular note brought in by non-formal education refers to the setting of the required teaching activities, outside the educational system. According to the specificity of the activities and the envisaged environment there are two categories: school-based activities (in the socio-professional environment) and out of school activities (in the socio-cultural environment: visits, trips, camps, etc.) (Blându, 2008; Bocoş and Jucan, 2008b; Cucoş, 2006). Non-formal education deals with the extension and completion of the knowledge horizon, by providing the participants with a properly organised environment where both spare time spending and entertaining are ensured, along with the practice and growth of skills and abilities (Cristea, 2006, p. 187).

Within the field of Geography there are various extracurricular teaching activities in which the instructive-educational side is associated with the recreational-entertaining one. According to the complexity degree, the objectives to be achieved, the means of organisation and the unfolding features, these activities define school tourism forms (Moşoarcă, 2005, p. 3), made materialised as in: walks, hiking, visits, tours, cycling trips, school trips, expeditions and school camps (Frant, 2003; Moşoarcă, 2005).

The extracurricular activity, usually taking place at county or regional level, which involves travel either on foot or using means of transportation and having tourism, recreation or education as main purposes (Blândul, 2008, p. 33), in pre-university environment is generically known as a school trip. Even though within the university environment this term is substituted by the phrase of scientific educational academic trip, it basically unfolds under similar circumstances; it takes place on a certain territory and it targets active preoccupations such as visiting, getting informed, studying and deepening the knowledge of some aspects related to the socio-economic and cultural reality.

School trips and academic trips require the existence of a geographic itinerary, "the road on which the trip takes place, with the specification of all the towns and villages and tourist attractions that will be visited throughout the trip" (Moşoarcă, 2005, p. 12), in the absence of which the mentioned extracurricular activities can be neither initiated, nor organised.

\section{MATERIAL AND METHOD}

\section{Research material}

The analysed materials from tourist (administrative) perspective are about information referring to: transportation services, accommodation services, catering services, recreational services (including passes to the tourist sights), the detailed route within the unfold of the school/academic trip programme, conceived to serve the educational purpose of the trip. 
The analysis of the proposed teaching activities involved the tasks for both students and undergraduates and the worksheets that all participants were asked to fill in during the itinerary. In brief, the students were required:

- to make an informative summary for one tourist attraction (which would serve to present the sight of their choice from the perspective of a tour guide, in front of their classmates);

- to fill in a worksheet, consisting of 5 fields, for each visited tourist attractions;

- to make a portfolio, containing the afore mentioned summary, the completed worksheets and a few relevant photos.

The undergraduates, were given more complex tasks, in their worksheets in which they were required:

- to elaborate an essay for one of the visited tourist sights (based on which they could present it in front of their colleagues as if they were tour guides);

- to fill in a worksheet, consisting of 9 fields, concerning actual management, development and tourism potential issues for each tourist attraction;

- to make a notebook for the trip, containing the required essay, all worksheets filled in and a few relevant photos.

\section{Method}

In this research, there were several stages:

- the design and analysis of a geographic-tourist itinerary, to the northwest of Romania, that would both fit a school trip and a scientific-educational academic trip;

- identifying and analysing the best way to combine all tourist services (transportation, accommodation, catering, entertainment, etc.) ;

- creating and analysing the trip planner for the proposed itinerary;

- conceiving and analysing differentiated teaching activities, according to the related educational level (pre-university/university);

- making and analysing the worksheets for these activities;

- comparing the two forms of non-formal education (the school trip and the scientific-educational academic trip) and highlighting their main similarities and differences.

We used the method of text analysis on the contents of the documents of both trips (itinerary, transportation services, accommodation, catering, and amenities), the students' portfolios and the undergraduates' trip notebooks. 
COMPARATIVE STUDY: SCHOOL TRIP AND SCIENTIFIC EDUCATIONAL ACADEMIC TRIP IN THE NORTH-WEST OF ROMANIA

\section{RESULTS}

\section{Tourist services and related information}

The itinerary was set for the North-Western Development Region, departing from and arriving to Cluj-Napoca. Having a total length of $800 \mathrm{~km}$, the itinerary integrated 35 tourist attractions, located within the following 30 localities: Sălicea, Petreştii de Jos, Turda, Buru, Iara, Muntele Băişorii, Plopi, Someşu Rece, Mărişel, Beliş, Răchiţele, Bologa, Valea Drăganului, Ciucea, Gâlgău Almaşului, Jibou, Baia Mare, Certeze, Săpânţa, Sighetu Marmaţiei, Vadu Izei, Ocna Şugatag, Budeşti, Sârbi, Bârsana, Vişeu de Sus, Moisei, Coşbuc, and Bonţida (Figure 1).

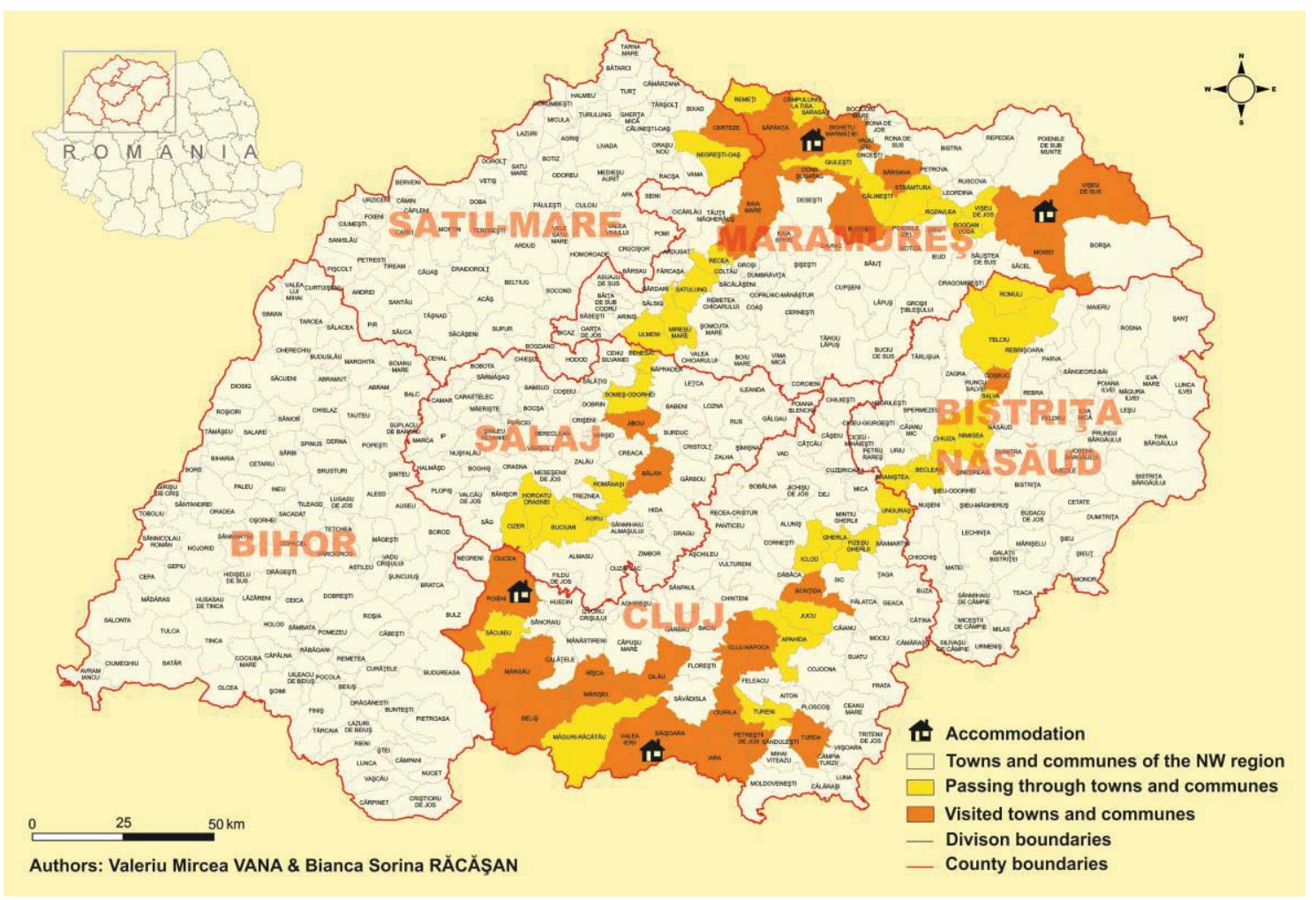

Fig. 1. The map of the proposed itinerary for extracurricular activities in the North-West of Romania

Having in mind a most accurate representation of the particularities of the tourist services that ensured the well unfolding of the trip, we put all pieces of information in a tabular format, grouped into four categories: transportation services (Table 1); accommodation services (Table 2); catering services (Table 3); amenities and entrance fees (Table 4). 
Table 1. Transportation services information

\begin{tabular}{|c|c|c|}
\hline & School trip & Academic trip \\
\hline Total no. of $\mathrm{km}$ & \multicolumn{2}{|r|}{800 km } \\
\hline $\begin{array}{l}\text { Transporting } \\
\text { company }\end{array}$ & \multicolumn{2}{|c|}{ S.C. TINUJA S.R.L. } \\
\hline Price $/ \mathrm{km}$ & \multicolumn{2}{|c|}{0.94 euro $/ \mathrm{km}$ or $4.165 \mathrm{lei} / \mathrm{km}$} \\
\hline Total price & \multicolumn{2}{|c|}{$\begin{array}{l}\text { 3,432 lei (transportation costs including parking fees: } 20 \text { lei/day = } \\
100 \text { lei/itinerary) }\end{array}$} \\
\hline $\begin{array}{l}\text { Means of } \\
\text { transportation }\end{array}$ & Setra Coach - 50 places & Setra Coach - 75 places \\
\hline No. of participants & $\begin{array}{l}50 \text { ( } 46 \text { students/paying } \\
\text { persons) }\end{array}$ & $\begin{array}{l}75 \text { ( } 70 \text { undergraduates/paying } \\
\text { persons) }\end{array}$ \\
\hline Price/person & 74,6 lei/person & $49 \mathrm{lei} /$ person \\
\hline
\end{tabular}

Table 2. Accommodation services information

\begin{tabular}{|c|c|c|c|c|c|c|c|}
\hline \multirow[t]{2}{*}{ No. } & \multirow{2}{*}{$\begin{array}{l}\text { Name of the } \\
\text { accommodation } \\
\text { unit (locality) }\end{array}$} & \multirow{2}{*}{$\begin{array}{l}\text { Number of } \\
\text { accommodation } \\
\text { places (rooms) }\end{array}$} & \multirow{2}{*}{$\begin{array}{l}\text { Type of } \\
\text { the room } \\
\text { and fee }\end{array}$} & \multicolumn{2}{|c|}{$\begin{array}{c}\text { School trip: } \\
50 \text { participants } \\
\text { (46 paying persons) }\end{array}$} & \multicolumn{2}{|c|}{$\begin{array}{c}\text { Academic trip: } \\
75 \text { participants } \\
\text { (70 paying persons) }\end{array}$} \\
\hline & & & & $\begin{array}{c}\text { Total } \\
\text { accommodation }\end{array}$ & $\begin{array}{c}\text { Total/ } \\
\text { person }\end{array}$ & $\begin{array}{c}\text { Total } \\
\text { accommodation }\end{array}$ & $\begin{array}{l}\text { Total/ } \\
\text { person }\end{array}$ \\
\hline $\begin{array}{c}\text { Day } \\
1\end{array}$ & $\begin{array}{l}\text { ALPIN } \\
\text { Hotel*** } \\
\text { (Muntele } \\
\text { Băişorii) }\end{array}$ & $\begin{array}{l}110 \text { places } \\
\text { (50 rooms) }\end{array}$ & $\begin{array}{l}\text { single: } \\
70 \text { lei } \\
\text { double: } \\
90 \text { lei } \\
\text { triple: } \\
120 \text { lei }\end{array}$ & $\begin{array}{l}\text { a) } 1 \text { single: } \\
70 \text { lei } \\
\text { b) } 17 \text { double: } \\
1,530 \text { lei } \\
5 \text { triple: } 600 \\
\text { lei } \\
\text { T: } \mathbf{2 , 2 0 0 ~ l e i ~}\end{array}$ & $\begin{array}{c}47.8 \\
\text { lei/ } \\
\text { person }\end{array}$ & $\begin{array}{l}\text { a) } 30 \text { double: } \\
2.700 \text { lei } \\
\text { b) } 5 \text { triple: } \\
600 \text { lei } \\
\text { T: } \mathbf{3 , 3 0 0 ~ l e i ~}\end{array}$ & $\begin{array}{c}47.1 \\
\text { lei/ } \\
\text { person }\end{array}$ \\
\hline $\begin{array}{c}\text { Day } \\
2\end{array}$ & $\begin{array}{l}\text { a) Casa } \\
\text { Bihoreană } \\
\text { Guest } \\
\text { House*** } \\
\text { b) Giurgiuma } \\
\text { n Guest } \\
\text { House*** } \\
\text { c) Andreea } \\
\text { Guest } \\
\text { House*** } \\
\text { (Valea } \\
\text { Drăganului) }\end{array}$ & $\begin{array}{l}\text { a) } 42 \text { places } \\
\text { ( } 19 \text { rooms) } \\
\text { b) } 26 \text { places } \\
\text { ( } 15 \text { rooms) } \\
\text { c) } 22 \text { places }\end{array}$ & $\begin{array}{l}\text { a) double } \\
: 70 \text { lei } \\
\text { b) entire } \\
\text { guest } \\
\text { house: } \\
550 \\
\text { lei/night } \\
\text { c) entire } \\
\text { guest } \\
\text { house: } \\
600 \\
\text { lei/night }\end{array}$ & $\begin{array}{l}\text { b) and c) } \\
\text { T:1,150 lei }\end{array}$ & $\begin{array}{l}25 \text { lei/ } \\
\text { person }\end{array}$ & $\begin{array}{l}\text { a) } 14 \\
\text { double: } 980 \\
\text { lei } \\
\text { b) } 550 \text { lei } \\
\text { c) } 600 \text { lei } \\
\text { T: } \mathbf{2 , 1 3 0 ~ l e i ~}\end{array}$ & $\begin{array}{l}30 \text { lei/ } \\
\text { person }\end{array}$ \\
\hline $\begin{array}{c}\text { Day } \\
3\end{array}$ & $\begin{array}{l}\text { a) Nova } \\
\text { Hotel*** } \\
\text { b) Buţi } \\
\text { Hotel*** } \\
\text { (Sighetu } \\
\text { Marmaţiei) }\end{array}$ & $\begin{array}{l}\text { a) } 56 \text { places } \\
\text { ( } 23 \text { rooms) } \\
\text { b) } 46 \text { places } \\
\text { ( } 18 \text { rooms } \\
\text { and } \\
\text { 3apartments) }\end{array}$ & $\begin{array}{l}\text { a) } \\
\text { double: } \\
140 \text { lei } \\
\text { b) } \\
\text { double: } \\
120 \text { lei }\end{array}$ & $\begin{array}{l}\text { a) } 25 \text { double: } \\
\text { T: } \mathbf{3 , 5 0 0 ~ l e i ~}\end{array}$ & $\begin{array}{l}76 \text { lei/ } \\
\text { person }\end{array}$ & $\begin{array}{l}\text { a) } 24 \\
\text { double: } \\
3.360 \text { lei } \\
\text { b) } 11 \\
\text { double: } \\
\text { 1.320 lei } \\
2 \text { triple: } 360 \\
\quad \text { lei } \\
\text { T: } \mathbf{5 , 0 4 0 ~ l e i ~}\end{array}$ & $\begin{array}{l}72 \text { lei/ } \\
\text { person }\end{array}$ \\
\hline $\begin{array}{c}\text { Day } \\
4\end{array}$ & $\begin{array}{l}\text { Gabriela } \\
\text { Hotel*** } \\
\text { (Vişeu de } \\
\text { Sus) }\end{array}$ & $\begin{array}{l}98 \text { places } \\
\text { ( } 50 \text { rooms) }\end{array}$ & $\begin{array}{l}\text { a) } \\
\text { double: } \\
\text { 130 lei } \\
\text { b) triple: } \\
170 \text { lei }\end{array}$ & $\begin{array}{c}18 \text { double: } \\
2.340 \text { lei } \\
5 \text { triple: } 850 \\
\text { lei } \\
\text { T: } \mathbf{3 , 1 9 0 ~ l e i ~}\end{array}$ & $\begin{array}{l}69 \text { lei/ } \\
\text { person }\end{array}$ & $\begin{array}{l}\text { a) } 30 \text { double: } \\
3.900 \text { lei } \\
\text { b) } 5 \text { triple: } \\
850 \text { lei } \\
\text { T: } \mathbf{4 , 7 5 0 ~ l e i ~}\end{array}$ & $\begin{array}{c}67.8 \\
\text { lei/ } \\
\text { person }\end{array}$ \\
\hline TOTAL & Price/person & - & - & 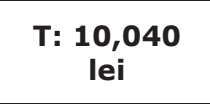 & $\begin{array}{c}217.8 \\
\text { lei/ } \\
\text { person }\end{array}$ & $\begin{array}{c}\text { T: } 15,220 \\
\text { lei }\end{array}$ & $\begin{array}{c}216.9 \\
\text { lei/ } \\
\text { person }\end{array}$ \\
\hline
\end{tabular}


Table 3. Catering services information

\begin{tabular}{|c|c|c|c|c|c|c|}
\hline \multirow[t]{2}{*}{ No. } & \multirow{2}{*}{$\begin{array}{l}\text { Type of } \\
\text { the meal } \\
\text { (locality) }\end{array}$} & \multirow{2}{*}{$\begin{array}{l}\text { Prices } \\
\text { (Name of the } \\
\text { catering unit) }\end{array}$} & \multicolumn{2}{|c|}{$\begin{array}{c}\text { School trip: } \\
\mathbf{5 0} \text { participants } \\
\text { (46 paying } \\
\text { persons) }\end{array}$} & \multicolumn{2}{|c|}{$\begin{array}{l}\text { Academic trip: } \\
75 \text { participants } \\
\text { (70 paying } \\
\text { persons) }\end{array}$} \\
\hline & & & $\begin{array}{l}\text { Total/ } \\
\text { meals }\end{array}$ & $\begin{array}{l}\text { Total/ } \\
\text { person }\end{array}$ & $\begin{array}{l}\text { Total/ } \\
\text { meals }\end{array}$ & $\begin{array}{l}\text { Total/ } \\
\text { Person }\end{array}$ \\
\hline \multirow{3}{*}{$\begin{array}{l}\text { Day } \\
1\end{array}$} & Breakfast & - & - & - & - & \\
\hline & $\begin{array}{l}\text { Lunch } \\
\text { (Turda) }\end{array}$ & $\begin{array}{l}13 \text { lei/pers. - Dana } \\
\text { Cafeteria }\end{array}$ & 650 lei & $\begin{array}{l}14.1 \\
\text { lei/pers. }\end{array}$ & 975 lei & $\begin{array}{l}13.9 \\
\text { lei/pers. }\end{array}$ \\
\hline & $\begin{array}{l}\text { Dinner } \\
\text { (Muntele } \\
\text { Băişorii) }\end{array}$ & $\begin{array}{l}20 \text { lei/pers. - Alpin } \\
\text { Hotel }\end{array}$ & $\begin{array}{l}1,000 \\
\text { lei }\end{array}$ & $\begin{array}{l}21.7 \\
\text { lei/pers. }\end{array}$ & $\begin{array}{l}1,500 \\
\text { lei }\end{array}$ & $\begin{array}{l}21.4 \\
\text { lei/pers. }\end{array}$ \\
\hline \multirow{4}{*}{$\begin{array}{l}\text { Day } \\
2\end{array}$} & $\begin{array}{l}\text { Breakfast } \\
\text { (Muntele } \\
\text { Băişorii) }\end{array}$ & $\begin{array}{l}15 \text { lei/pers. - Alpin } \\
\text { Hotel }\end{array}$ & 750 lei & $\begin{array}{l}16.3 \\
\text { lei/pers. }\end{array}$ & $\begin{array}{l}1,125 \\
\text { lei }\end{array}$ & $\begin{array}{l}16 \\
\text { lei/pers. }\end{array}$ \\
\hline & $\begin{array}{l}\text { Lunch } \\
\text { (Mărişel) }\end{array}$ & $\begin{array}{l}20 \text { lei/pers. - Moţilor } \\
\text { Chalet }\end{array}$ & $\begin{array}{l}1,000 \\
\text { lei }\end{array}$ & $\begin{array}{l}21.7 \\
\text { lei/pers. }\end{array}$ & $\begin{array}{l}1,500 \\
\text { lei }\end{array}$ & $\begin{array}{l}21.4 \\
\text { lei/pers. }\end{array}$ \\
\hline & $\begin{array}{l}\text { *Snack } \\
\text { (Răchiţele) }\end{array}$ & $\begin{array}{l}5 \text { lei/pers. - Şuşman } \\
\text { Guest House }\end{array}$ & 250 lei & $\begin{array}{l}5.4 \\
\text { lei/pers. }\end{array}$ & 375 lei & $\begin{array}{l}5.3 \\
\text { lei/pers. }\end{array}$ \\
\hline & $\begin{array}{l}\text { Dinner } \\
\text { (Valea } \\
\text { Drăganului) }\end{array}$ & $\begin{array}{l}12 \text { lei/pers. - } \\
\text { Giurgiuman Guest } \\
\text { House }\end{array}$ & 600 lei & $\begin{array}{l}13 \\
\text { lei/pers. }\end{array}$ & 900 lei & $\begin{array}{l}12.8 \\
\text { lei/pers. }\end{array}$ \\
\hline \multirow{3}{*}{$\begin{array}{l}\text { Day } \\
3\end{array}$} & $\begin{array}{l}\text { Breakfast } \\
\text { (Valea } \\
\text { Drăganului) }\end{array}$ & $\begin{array}{l}8 \text { lei/pers. - } \\
\text { Giurgiuman Guest } \\
\text { House }\end{array}$ & 400 lei & $\begin{array}{l}8.6 \\
\text { lei/pers. }\end{array}$ & 600 lei & $\begin{array}{l}8.5 \\
\text { lei/pers. }\end{array}$ \\
\hline & $\begin{array}{l}\text { Lunch } \\
\text { (Jibou) }\end{array}$ & $\begin{array}{l}15 \text { lei/pers. - Alex } \\
\text { Complex }\end{array}$ & 750 lei & $\begin{array}{l}16.3 \\
\text { lei/pers. }\end{array}$ & $\begin{array}{l}1,125 \\
\text { lei }\end{array}$ & $\begin{array}{l}16 \\
\text { lei/pers. }\end{array}$ \\
\hline & $\begin{array}{l}\text { Dinner } \\
\text { (Sighetu } \\
\text { Marmaţiei) }\end{array}$ & $\begin{array}{l}30 \text { lei/pers. - Nova } \\
\text { and Buţi Hotels }\end{array}$ & $\begin{array}{l}1,500 \\
\text { lei }\end{array}$ & $\begin{array}{l}32.6 \\
\text { lei/pers. }\end{array}$ & $\begin{array}{l}2,250 \\
\text { lei }\end{array}$ & $\begin{array}{l}32.1 \\
\text { lei/pers. }\end{array}$ \\
\hline \multirow{3}{*}{$\begin{array}{l}\text { Day } \\
4\end{array}$} & $\begin{array}{l}\text { Breakfast } \\
\text { (Sighetu } \\
\text { Marmaţiei) }\end{array}$ & $\begin{array}{l}\text { Part of the } \\
\text { accommodation } \\
\text { package - Nova and } \\
\text { Buţi Hotels }\end{array}$ & - & - & - & - \\
\hline & $\begin{array}{l}\text { Lunch } \\
\text { (Ocna } \\
\text { Şugatag) }\end{array}$ & $\begin{array}{l}35 \text { lei/pers. - Popasul } \\
\text { din Deal Guest House }\end{array}$ & $\begin{array}{l}1,750 \\
\text { lei }\end{array}$ & $\begin{array}{l}38 \\
\text { lei/pers. }\end{array}$ & $\begin{array}{l}2,625 \\
\text { lei }\end{array}$ & $\begin{array}{l}37.5 \\
\text { lei/pers. }\end{array}$ \\
\hline & $\begin{array}{l}\text { Dinner } \\
\text { (Vişeu de } \\
\text { Sus) }\end{array}$ & $\begin{array}{l}50 \text { lei/pers. - Gabriela } \\
\text { Hotel }\end{array}$ & $\begin{array}{l}2,500 \\
\text { lei }\end{array}$ & $\begin{array}{l}54.3 \\
\text { lei/pers. }\end{array}$ & $\begin{array}{l}3,750 \\
\text { lei }\end{array}$ & $\begin{array}{l}53.5 \\
\text { lei/pers. }\end{array}$ \\
\hline \multirow{3}{*}{$\begin{array}{l}\text { Day } \\
5\end{array}$} & $\begin{array}{l}\text { Breakfast } \\
\text { (Vişeu de } \\
\text { Sus) }\end{array}$ & $\begin{array}{l}\text { Part of the } \\
\text { accommodation } \\
\text { package - Gabriela } \\
\text { Hotel }\end{array}$ & - & - & - & - \\
\hline & $\begin{array}{l}\text { *Snack } \\
\text { (Moisei) }\end{array}$ & $\begin{array}{l}7 \text { lei/pers. - Gabriela } \\
\text { Hotel }\end{array}$ & 350 lei & $\begin{array}{l}7.6 \\
\text { lei/pers. }\end{array}$ & 525 lei & $\begin{array}{l}7.5 \\
\text { lei/pers. }\end{array}$ \\
\hline & Dinner & - & - & - & - & - \\
\hline TOTAL & $\begin{array}{l}\text { Price/ } \\
\text { person }\end{array}$ & - & $\begin{array}{l}\text { T: } \\
11,500 \\
\text { lei }\end{array}$ & $\begin{array}{l}249.6 \\
\text { lei/pers. }\end{array}$ & $\begin{array}{l}\text { T: } \\
17,250 \\
\text { lei }\end{array}$ & $\begin{array}{l}245.9 \\
\text { lei/pers. }\end{array}$ \\
\hline
\end{tabular}


Table 4. Recreational activities and entrance fees information

\begin{tabular}{|c|c|c|c|c|c|c|}
\hline \multirow[t]{2}{*}{ No. } & \multirow[t]{2}{*}{$\begin{array}{l}\text { Name of the } \\
\text { Objective }\end{array}$} & \multirow[t]{2}{*}{$\begin{array}{l}\text { Entrance } \\
\text { fees }\end{array}$} & \multicolumn{2}{|c|}{$\begin{array}{c}\text { School trip: } \\
50 \text { participants } \\
\text { (46 paying persons) }\end{array}$} & \multicolumn{2}{|c|}{$\begin{array}{l}\text { Academic trip: } \\
75 \text { participants } \\
\text { ( } 70 \text { paying persons) }\end{array}$} \\
\hline & & & Total & Total/pers. & Total & Total/pers. \\
\hline \multirow{3}{*}{ Day 1} & $\begin{array}{l}\text { "Moara de } \\
\text { Vânt" Zoo- } \\
\text { park }\end{array}$ & 6 lei/pers. & 294 lei & $\begin{array}{l}6.3 \\
\text { lei/pers. }\end{array}$ & 444 lei & $\begin{array}{l}6.3 \\
\text { lei/pers. }\end{array}$ \\
\hline & Turda Gorges & 3 lei/pers. & 147 lei & $\begin{array}{l}3.2 \\
\text { lei/pers. }\end{array}$ & 222 lei & $\begin{array}{l}3.2 \\
\text { lei/pers. }\end{array}$ \\
\hline & $\begin{array}{l}\text { Turda Salt } \\
\text { Mine }\end{array}$ & 10 lei/pers. & 490 lei & $\begin{array}{l}10.6 \\
\text { lei/pers. }\end{array}$ & 740 lei & $\begin{array}{l}10.6 \\
\text { lei/pers. }\end{array}$ \\
\hline \multirow{4}{*}{ Day 3} & $\begin{array}{l}\text { "Octavian } \\
\text { Goga" } \\
\text { Memorial } \\
\text { Museum } \\
\end{array}$ & 5 lei/pers. & 245 lei & $\begin{array}{l}5.3 \\
\text { lei/pers. }\end{array}$ & 370 lei & $\begin{array}{l}5.3 \\
\text { lei/pers. }\end{array}$ \\
\hline & $\begin{array}{l}\text { Jibou } \\
\text { Botanical } \\
\text { Garden }\end{array}$ & 3 lei/pers. & 147 lei & $\begin{array}{l}3.2 \\
\text { lei/pers. }\end{array}$ & 222 lei & $\begin{array}{l}3.2 \\
\text { lei/pers. }\end{array}$ \\
\hline & $\begin{array}{l}\text { Baia Mare } \\
\text { Museum of } \\
\text { Mineralogy }\end{array}$ & 3 lei/pers. & 147 lei & $\begin{array}{l}3.2 \\
\text { lei/pers. }\end{array}$ & 222 lei & $\begin{array}{l}3.2 \\
\text { lei/pers. }\end{array}$ \\
\hline & $\begin{array}{l}\text { The Jolly } \\
\text { Cemetery of } \\
\text { Săpânţa }\end{array}$ & 2 lei/pers. & 98 lei & $\begin{array}{l}2.1 \\
\text { lei/pers. }\end{array}$ & 148 lei & $\begin{array}{l}2.1 \\
\text { lei/pers. }\end{array}$ \\
\hline \multirow{3}{*}{ Day 4} & $\begin{array}{l}\text { The Memorial } \\
\text { Prison of } \\
\text { Sighet }\end{array}$ & 3 lei/pers. & 147 lei & $\begin{array}{l}3.2 \\
\text { lei/pers. }\end{array}$ & 222 lei & $\begin{array}{l}3.2 \\
\text { lei/pers. }\end{array}$ \\
\hline & $\begin{array}{l}\text { Maramureş } \\
\text { Village } \\
\text { Museum } \\
\end{array}$ & 3 lei/pers. & 147 lei & $\begin{array}{l}3.2 \\
\text { lei/pers. }\end{array}$ & 222 lei & $\begin{array}{l}3.2 \\
\text { lei/pers. }\end{array}$ \\
\hline & $\begin{array}{l}\text { Bârsana } \\
\text { Monastery }\end{array}$ & 1 leu/pers. & 49 lei & $\begin{array}{l}1.1 \\
\text { lei/pers. }\end{array}$ & 74 lei & $\begin{array}{l}1.1 \\
\text { lei/pers. }\end{array}$ \\
\hline \multirow{3}{*}{ Day 5} & $\begin{array}{l}\text { Amusement } \\
\text { Transportation } \\
\text { Mocăniţa }\end{array}$ & $\begin{array}{l}34.2^{*} \\
\text { lei/pers. }\end{array}$ & $\begin{array}{l}1.675 \\
\text { lei }\end{array}$ & $\begin{array}{l}36.4 \\
\text { lei/pers. }\end{array}$ & $\begin{array}{l}2.530 \\
\text { lei }\end{array}$ & $\begin{array}{l}36.2 \\
\text { lei/pers. }\end{array}$ \\
\hline & $\begin{array}{l}\text { "George } \\
\text { Coşbuc" } \\
\text { Memorial } \\
\text { Museum }\end{array}$ & 3 lei/pers. & 147 lei & $\begin{array}{l}3.2 \\
\text { lei/pers. }\end{array}$ & 222 lei & $\begin{array}{l}3.2 \\
\text { lei/pers. }\end{array}$ \\
\hline & $\begin{array}{l}\text { The Banffy } \\
\text { Castle }\end{array}$ & 3 lei/pers. & 147 lei & $\begin{array}{l}3.2 \\
\text { lei/pers. }\end{array}$ & 222 lei & $\begin{array}{l}3.2 \\
\text { lei/pers. }\end{array}$ \\
\hline TOTAL & Price/person & & $\begin{array}{l}\text { T: } \\
3,880 \\
\text { lei }\end{array}$ & $\begin{array}{l}84.2 \\
\text { lei/pers. }\end{array}$ & $\begin{array}{l}\text { T: } \\
5,860 \\
\text { lei }\end{array}$ & 84 lei/pers. \\
\hline
\end{tabular}

After adding up the costs of all the services of transportation, accommodation, catering and entrance fees, the final price was set for the trips in the North-West of Romania, at the end of April, on a duration of 5 days (Table 5). 
Table 5. Total costs of the school and of the scientific educational trips

\begin{tabular}{|c|c|c|}
\hline $\begin{array}{l}\text { Tourist services } \\
\text { information }\end{array}$ & $\begin{array}{c}\text { School trip } \\
\text { (Prices) }\end{array}$ & $\begin{array}{c}\text { Academic trip } \\
\text { (Prices) }\end{array}$ \\
\hline No. of participants & $\begin{array}{l}50 \text { ( } 46 \text { students/ paying } \\
\text { persons) }\end{array}$ & $\begin{array}{l}75 \text { ( } 70 \text { undergraduates/paying } \\
\text { persons) }\end{array}$ \\
\hline Transportation & $74.6 \mathrm{lei} /$ person & $49 \mathrm{lei} /$ person \\
\hline Accommodation & 217.8 lei/person & 216.9 lei/person \\
\hline Catering & 249.6 lei/person & 245.9 lei/person \\
\hline Entrance fees & 84.2 lei/person & 84 lei/person \\
\hline Total & (626.2 lei/person) & (595.8 lei/person) \\
\hline Final price & $625 \mathrm{lei} /$ person & 585 lei/person \\
\hline
\end{tabular}

Considering all these administrative aspects regarding the organisation, we came to the following trip planner and programme for the 5 days of itinerary (Table 6 ).

Table 6. Trip planner

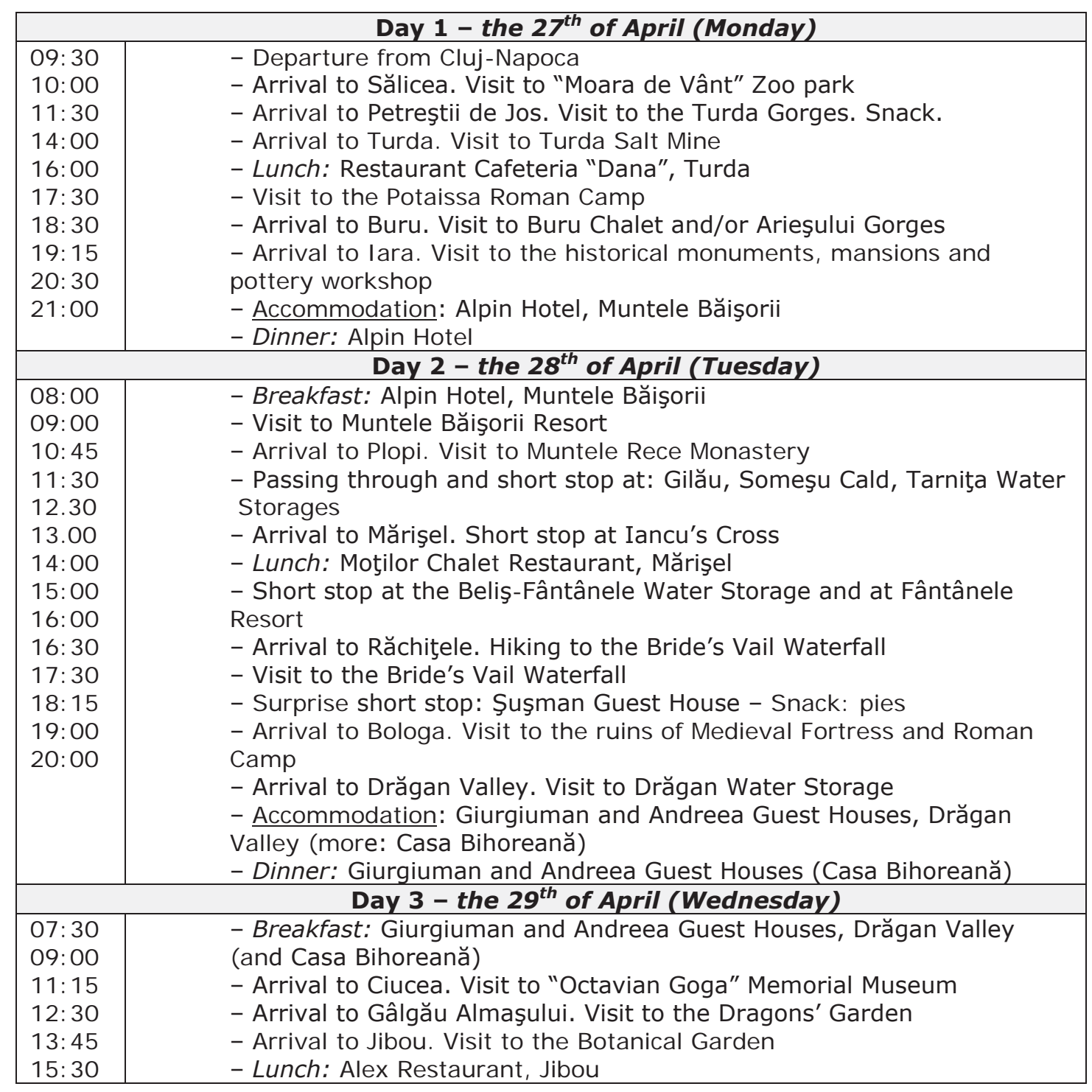




\begin{tabular}{|c|c|}
\hline $\begin{array}{l}17: 30 \\
18: 30 \\
19: 30 \\
20: 30 \\
21: 00\end{array}$ & $\begin{array}{l}\text { - Arrival to Baia Mare. Visit to the County Museum of Mineralogy } \\
\text { - Passing through Certeze. Short stop } \\
\text { - Arrival to Săpânţa. Visit to the Jolly Cemetery (craftsmen's workshop) } \\
\text { - Visit to the Săpânţa-Peri Monastery } \\
\text { - Accommodation: Nova and Buţi Hotels, Sighetu Marmaţiei } \\
\text { - Dinner: Nova and Buţi Hotels }\end{array}$ \\
\hline \multicolumn{2}{|r|}{ Day $4-$ the $30^{\text {th }}$ of April (Thursday) } \\
\hline $\begin{array}{l}\text { 08:00 } \\
09: 30 \\
11: 00 \\
12: 45 \\
14: 00 \\
15: 15 \\
16: 00 \\
17: 15 \\
18: 15 \\
20: 30 \\
21: 00\end{array}$ & $\begin{array}{l}\text { - Breakfast: Nova and Buţi Hotels, Sighetu Marmaţiei } \\
\text { - Visit to the Memorial Prison at Sighet } \\
\text { - Visit to the Maramureş Village Museum } \\
\text { - Arrival to Vadu Izei. Visit to a complex of traditional technical } \\
\text { installations } \\
\text { - Lunch: Popasul din Deal Guest House Restaurant, Ocna Şugatag } \\
\text { - Visit to the Ocna Şugatag Resort } \\
\text { - Arrival to Budeşti. Visit to the wooden church belonging to the } \\
\text { UNESCO patrimony } \\
\text { - Arrival to Sârbi. Visit to the workshop of a hat maker } \\
\text { - Arrival to Bârsana. Visit to the Bârsana Monastery } \\
\text { - Accommodation: Gabriela Hotel, Vişeu de Sus } \\
\text { - An evening in Maramureş: (traditional dinner + tourist animation) }\end{array}$ \\
\hline \multicolumn{2}{|r|}{ Day 5 - the $1^{\text {st }}$ of May (Friday) } \\
\hline $\begin{array}{l}07: 30 \\
09: 00 \\
14: 45 \\
16: 30 \\
19: 00 \\
21: 30\end{array}$ & $\begin{array}{l}\text { - Breakfast: Gabriela Hotel, Vişeu de Sus } \\
\text { - Amusement Transportation: with the narrow railway on the Vaser } \\
\text { Valley } \\
\text { - Arrival to Moisei. Short stop to the Martyrs' Monument. Snack } \\
\text { - Arrival to Coşbuc. Visit to "George Coşbuc" Memorial Museum } \\
\text { - Arrival to Bontida. Visit to Banffy Castle } \\
\text { - Arrival to Cluj-Napoca }\end{array}$ \\
\hline
\end{tabular}

\section{Organised activities for students and undergraduates}

Both students and undergraduates were required to solve a series of tasks, presented in Table 7.

Table 7. The students' and undergraduates' activities (teaching plan)

\begin{tabular}{|c|c|}
\hline School trip & Academic trip \\
\hline \multicolumn{2}{|c|}{ Stage 1 (preparing the activities, before the field trip) } \\
\hline $\begin{array}{l}\text { - informing the students } \\
\text { about the activities they are } \\
\text { going to undergo in the } \\
\text { three stages of the trip } \\
\text { (previous to, during, and } \\
\text { after the field trip) } \\
\text { - informing them about the } \\
\text { itinerary and about the } \\
\text { tourist attractions } \\
\text { - establishing the tourist } \\
\text { attraction each student is } \\
\text { going to present, by drawing } \\
\text { lots } \\
\text { - presenting the tasks: } \\
\text { 1. writing an informational } \\
\text { summary for the tourist } \\
\text { attraction that has been } \\
\text { drawn }\end{array}$ & $\begin{array}{l}\text { - informing the undergraduates on the activities they are } \\
\text { going to undergo in the three stages of the trip (previous } \\
\text { to, during, and after the field trip) } \\
\text { - informing them about the itinerary and on the tourist } \\
\text { attractions } \\
\text { - establishing the tourist attraction which the } \\
\text { undergraduates, in pairs, are going to present in detail, by } \\
\text { drawing lots } \\
\text { - presenting the tasks: } \\
\text { 1. identifying general information about the proposed } \\
\text { itinerary (localities, landmarks, accommodation), } \\
\text { information available in the trip planner } \\
\text { 2. writing down the information within the trip notebook } \\
\text { 3. researching web sources about the visibility of the } \\
\text { tourist landmark (the existence of own sites, forum, } \\
\text { Facebook page etc.) } \\
\text { 4. writing down the information on the worksheets } \\
\text { belonging to each tourist attraction }\end{array}$ \\
\hline
\end{tabular}




\section{COMPARATIVE STUDY: SCHOOL TRIP AND SCIENTIFIC EDUCATIONAL ACADEMIC TRIP IN THE NORTH-WEST OF ROMANIA}

\begin{tabular}{|c|c|}
\hline $\begin{array}{l}\text { 2. informing them about the } \\
\text { "Junior Tour Guide: Best } \\
\text { Presentation" Contest } \\
\text { 3. familiarising students with } \\
\text { the tasks on the worksheet } \\
\text { (Figure 1), that need to be } \\
\text { filled in on the spot for each } \\
\text { tourist attraction } \\
\text { 4. informing them about the } \\
\text { evaluation stage of the } \\
\text { contents of the portfolio they } \\
\text { need to present at the end } \\
\text { of the trip }\end{array}$ & $\begin{array}{l}\text { 5. writing an essay about the drawn tourist attraction } \\
\text { 6. informing the undergraduates about "The Best Tour } \\
\text { Guide" Contest } \\
\text { 7. familiarising undergraduates with the tasks in the } \\
\text { worksheets (Figure 2), that need to be filled in, on the } \\
\text { spot, for each landmark } \\
\text { 8. getting them informed about the evaluation and the } \\
\text { contents of the trip notebook }\end{array}$ \\
\hline $\begin{array}{l}\quad \text { Stage } 2 \text { (tudents' tasks: } \\
\text { 1. making the in situ } \\
\text { presentations, relying on the } \\
\text { previously prepared } \\
\text { informational summary, } \\
\text { written by each student } \\
\text { 2. filling in the worksheets } \\
\text { for each tourist attraction } \\
\text { considering: } \\
\text { 2.1. the identification of at } \\
\text { least } 2 \text { strengths and } 2 \\
\text { weaknesses of the visited } \\
\text { sight } \\
2.2 \text {. the evaluation of each } \\
\text { tourist attraction using a } 1 \\
\text { to } 10 \text { score scale (based on } \\
\text { personal experience, } \\
\text { expectations, etc.) } \\
2.3 \text {. an evaluation for the } \\
\text { tour guide student's } \\
\text { presentation using the same } \\
\text { scoring ( } 1 \text { to } 10 \text { ) } \\
2.4 \text {. the classification of the } \\
\text { tourist attractions, based on } \\
\text { their affiliation to one of the } \\
\text { following categories: relief, } \\
\text { climate, hydrography, } \\
\text { vegetation and fauna, } \\
\text { historical and archaeological } \\
\text { remains, religious } \\
\text { constructions, cultural } \\
\text { buildings and monuments, } \\
\text { ethnography and folklore } \\
2.5 \text {. the comparison } \\
\text { between the visited tourist } \\
\text { sight and the other ones } \\
\text { belonging to the same } \\
\text { category (resorts, } \\
\text { monasteries, water } \\
\text { storages, museums, } \\
\text { craftsmen's workshops, } \\
\text { camps, etc.) }\end{array}$ & $\begin{array}{l}\text { Undergraduates' tasks: } \\
\text { 1. making the in situ presentations, relying on the } \\
\text { previously prepared essay, written by each pair of } \\
\text { undergraduates designated by the professor } \\
\text { 2. filling in the worksheets for each tourist attraction } \\
\text { considering: } \\
\text { 2.1. the analysis of the current status of the visited } \\
\text { attraction, in terms of tourist management and } \\
\text { development (Landmarks: presence/absence of indicators, } \\
\text { informational materials and souvenirs buying possibilities, } \\
\text { tourist services (guide/person capable to answer the } \\
\text { tourists' questions), commercial spaces, sanitary } \\
\text { equipment, parking, visitation program, conservation } \\
\text { status, etc.) } \\
\text { 2.2. the identification of at least } 3 \text { strengths and } 3 \\
\text { weaknesses of the management and development of the } \\
\text { visited tourist attractions (according to the afore mentioned } \\
\text { landmarks) } \\
\text { 2.3. the enunciation of proposals for the valorisation of the } \\
\text { visited tourist attraction (taking into account the afore } \\
\text { mentioned landmarks) } \\
\text { 2.4. the comparison of the virtual image with the real one } \\
\text { (taking into account the afore mentioned landmarks) } \\
\text { 2.5. the evaluation of each tourist attraction using a } 1 \text { to } \\
\text { 10 score scale (based on personal experience, } \\
\text { expectations) } \\
\text { 2.6. the evaluation of the undergraduate pair's } \\
\text { presentation, using the same score scale (1 to 10) } \\
2.7 \text {. the classification of the tourist attractions, according to } \\
\text { the natural resources category to which it belongs } \\
\text { (morphologic, climatic, hydro-geographic or biogeographic) } \\
\text { or whether it is man-made patrimony (historical buildings, } \\
\text { religious buildings, cultural buildings, economical buildings } \\
\text { with tourist functions, ethnographic resources) } \\
\text { 2.8. the affiliation of each visited attraction to a tourism } \\
\text { form category (mountain tourism, rural tourism, cultural } \\
\text { tourism, climatic tourism, religious tourism) } \\
\text { 2.9. the comparison between the visited tourist sight and } \\
\text { the other ones belonging to the same category (resorts, } \\
\text { monasteries, water storages, museums, craftsmen's } \\
\text { workshops, camps, etc.) }\end{array}$ \\
\hline
\end{tabular}




\begin{tabular}{|c|c|}
\hline \multicolumn{2}{|c|}{ Stage 3 (evaluating participants' results and activities, after the field trip) } \\
\hline $\begin{array}{l}\text { - handing in the } \\
\text { portfolio containing: } \\
\text { a. information about the } \\
\text { itinerary (the localities that } \\
\text { were visited, names of the } \\
\text { visited tourist attractions); } \\
\text { b. the informative summary } \\
\text { for the tourist sight, "The } \\
\text { Junior Tour Guide: best } \\
\text { presentation" Contest; } \\
\text { c. punctual observations of } \\
\text { the other tourist attractions } \\
\text { (written down during the } \\
\text { colleagues' presentations); } \\
\text { d. the worksheets, } \\
\text { completely filled in, } \\
\text { individually, for each tourist } \\
\text { sight; } \\
\text { e. the evaluation for the } \\
\text { colleagues' presentations } \\
\text { and for the visited sights; } \\
\text { f. the classification of the } \\
\text { tourist sights; } \\
\text { g. the comparison between } \\
\text { the tourist attractions } \\
\text { belonging to the same } \\
\text { category; } \\
\text { h. other related } \\
\text { informational materials: } \\
\text { brochures, leaflets, fliers } \\
\text { etc. } \\
\text { i. } 7 \text { personal photos (at } \\
\text { least) }\end{array}$ & $\begin{array}{l}\text { - handing in the trip notebook filled in with the } \\
\text { required: } \\
\text { a. information about the itinerary (the localities that were } \\
\text { visited, names of the visited tourist attractions); } \\
\text { b. information about the visibility of the tourist sight to be } \\
\text { found in online entertainment; } \\
\text { c. the essay prepared for the tour guide moment and "The } \\
\text { Best Tour Guide" Contest; } \\
\text { d. punctual observations of the other tourist attractions } \\
\text { (written down during the colleagues' presentations); } \\
\text { e. analysis of the current status of the visited sights of its } \\
\text { tourist management and development (strengths, } \\
\text { weaknesses); } \\
\text { f. proposals for a proper valorisation of the resources' } \\
\text { tourism potential; } \\
\text { g. evaluation for the colleagues' presentations and for the } \\
\text { visited sights; } \\
\text { h. classification of the tourist attractions; } \\
\text { i. affiliation of the visited sight to a certain form of tourism; } \\
\text { j. comparison between the tourist attractions belonging to } \\
\text { the same category; } \\
\text { k. other informational materials: brochures, leaflets, fliers } \\
\text { etc. } \\
\text { l. } 12 \text { personal photos (at least) }\end{array}$ \\
\hline
\end{tabular}

The worksheets include the tasks for both students (Figure 2) and undergraduates (Figure 3).

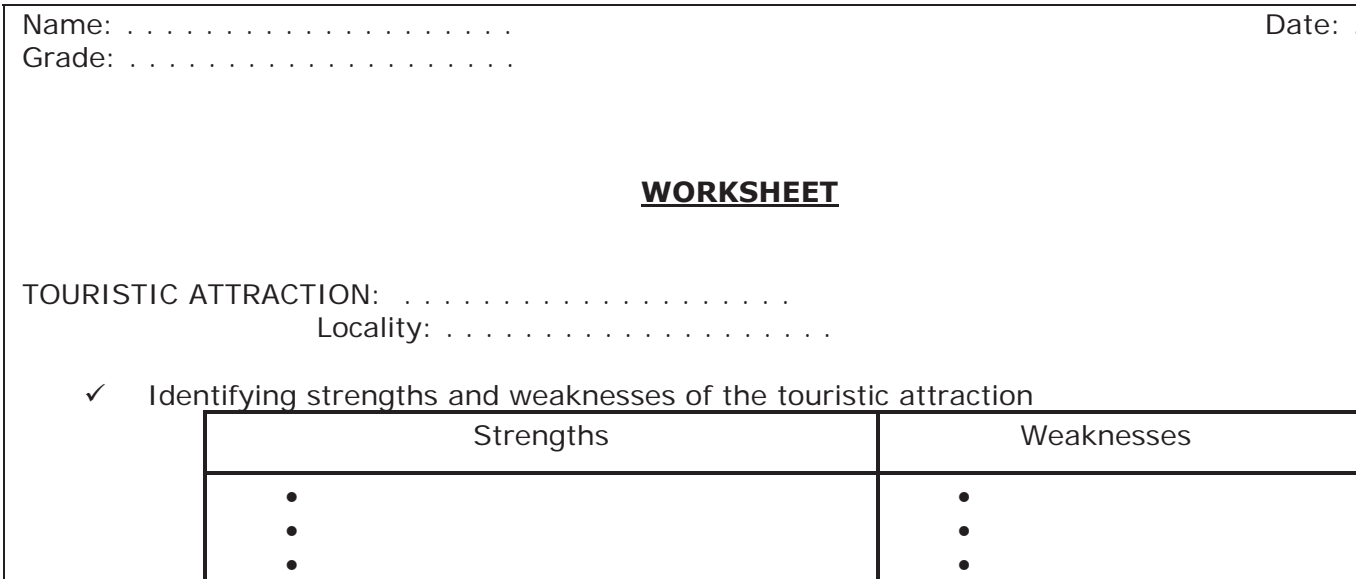

$\checkmark \quad$ Evaluation point score for the visited touristic attraction: . pt.

$\checkmark \quad$ Evaluation point score for colleague's presentation: pt. 
COMPARATIVE STUDY: SCHOOL TRIP AND SCIENTIFIC EDUCATIONAL ACADEMIC TRIP IN THE NORTH-WEST OF ROMANIA

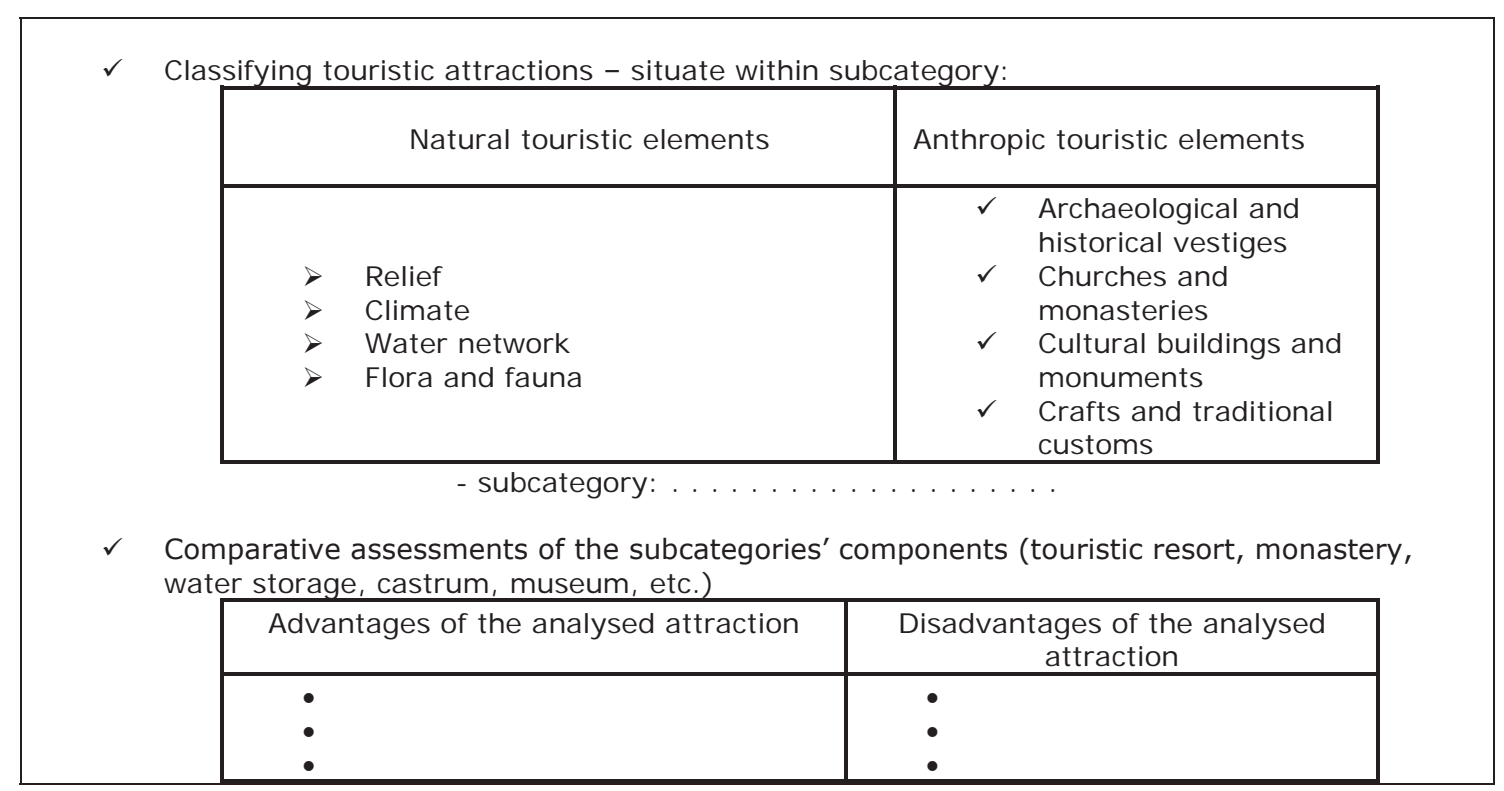

Fig. 2. The worksheet from students' portfolios

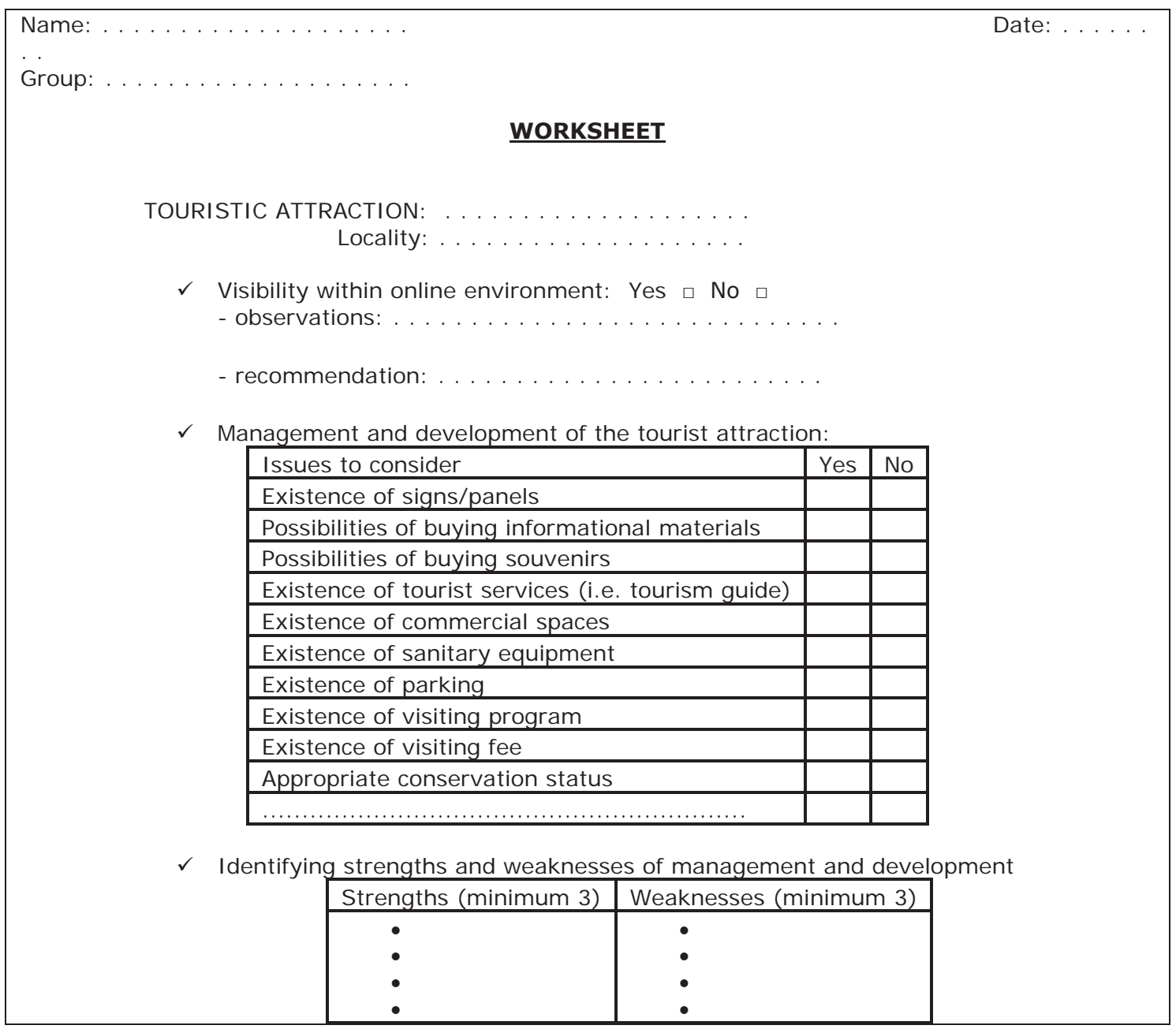




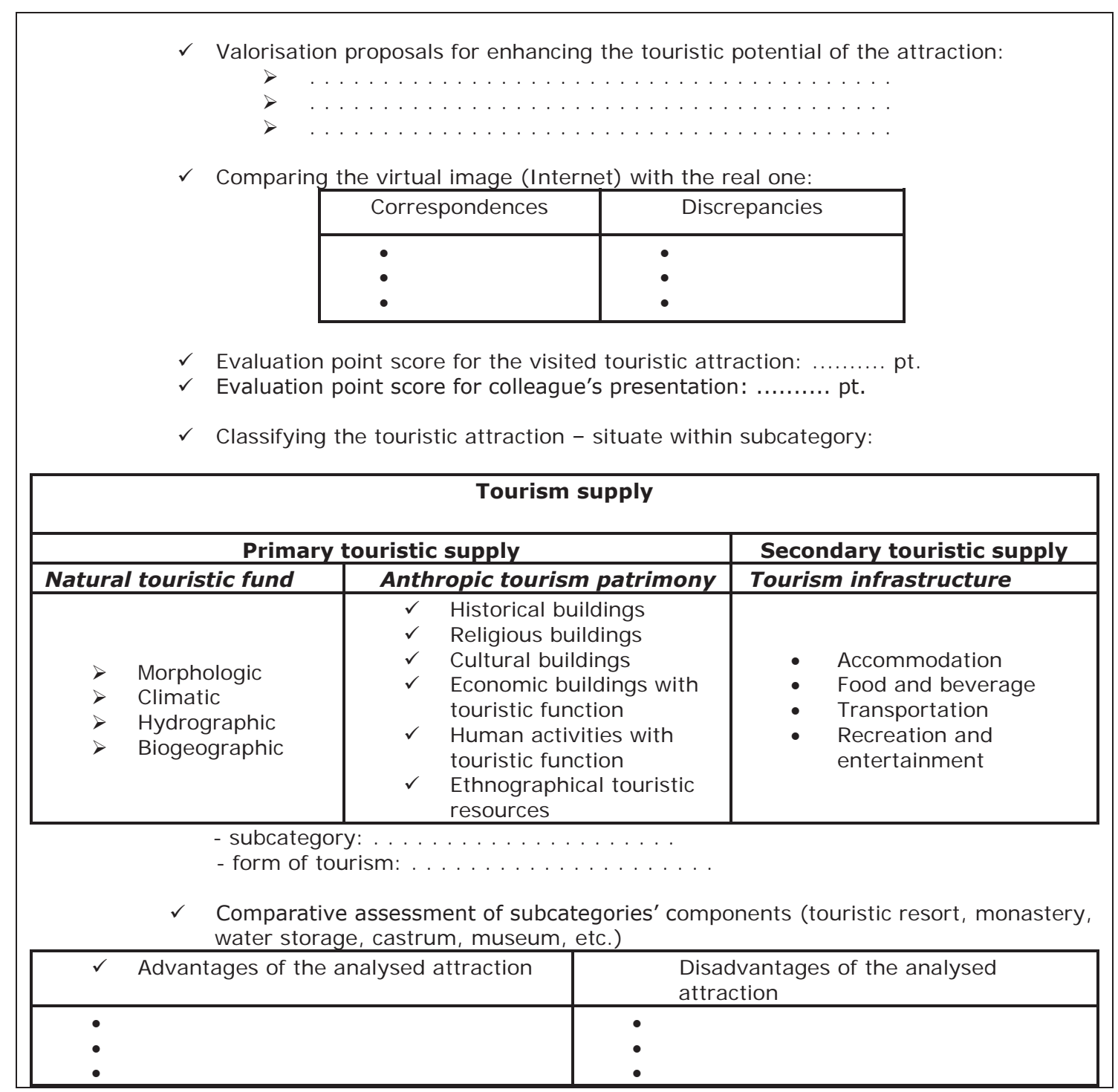

Fig. 3. The worksheet from undergraduates' trip notebook

\section{Students' and undergraduates' results}

At the end of the trip, the 46 students handed in their portfolios consisting of 35 worksheets filled in separately for each tourist sight, an informative summary for the attraction presented as a tour guide, and 7 photos.

The 70 undergraduates handed in, at the end of the trip, their trip notebooks containing the 35 worksheets with the analysis of each tourist attraction, the essay, and the 12 most suggestive photos made in the field. 


\section{DISCUSSION}

\section{Analysis of the touristic information and services}

The proposed itinerary is for a visit to Romania's North-Western Development Region, which has Cluj-Napoca as main city. The itinerary stretches on $800 \mathrm{~km}$, is includes 5 days and it was conceived to include localities and resources with tourist function. It integrated 35 tourist attractions located within 30 localities (Figure 1). They travelled $185 \mathrm{~km}$, on average, per day, visiting 6 sights daily, enough to allow the students/undergraduates to fill in the worksheets in due time, while also keeping them focused on the objectives.

The transportation services and company were contracted based on the routes they activated on regularly, on the capacity of the vehicles they possessed and on the best quality versus price relation. A coach of 50 places was chosen for the trip, in order to accommodate the entire number of 46 students and 4 teachers. A coach of 75 places was chosen for the academic trip, for the 70 undergraduates and the 5 accompanying professors. If a fee of 0.94 euros ( $4.165 \mathrm{lei} / \mathrm{km}$ ) was applied per $\mathrm{km}$ that amounted to a total of 3.400 lei for the entire $800 \mathrm{~km}$ of the itinerary. However, due to the smaller number of students, the transportation costs for the school trip (74.6 lei/person) were higher than those for the academic trip (49 lei/person) (Table 1 ).

Regarding the accommodation services, the best offers were studied and selected, considering their capacity to accommodate such high numbers, their proximity to the envisaged tourist attractions and the best quality versus price relation. The costs did not vary greatly. Considering the fact that a single room was 70 lei and a triple room was 170 lei, each participant paid approximatively 217 lei for the 4 nights of accommodation (Table 2).

With respect to the catering services, the same characteristics as in the case of accommodation were taken into account, especially the capacity to accommodate the large number of participants and their proximity to the envisaged sights and the following stop. The participants paid approximatively 247 lei/person for 3 meals a day. Breakfast fees were included within the accommodation price and dinner, although paid for separately, was served in the restaurants of the accommodation units (Table 3). The average time allotted for serving the meals was of $1 \mathrm{~h} 30$ minutes, with a minimum for the snacks served in Turda Gorges, Răchitele and Moisei, and a maximum time allotted for the traditional Maramureş evening organised in Vişeu de Sus, in the last night of the trip.

As for the cost of the entertainment services, a third of the visited sights had entrance fees varying from 1 to $10 \mathrm{lei} /$ person (the Turda Salt Mine, "Octavian Goga" Museum, Jibou Botanical Garden, the Jolly Cemetery of Săpânţa, the Banffy Castle, etc.). Seven of these tourist attractions had a very reasonable fee of 3 lei/person. The visit to the Bârsana Monastery and 
to the museum within was the cheapest of all ( 1 leu/person). The most expensive and most interesting form of entertainment was definitely the Mocaniţa ride on the Vaser Valley (36 lei/pers.), with groups larger than 15 persons, benefitting from a $10 \%$ discount. Thus, out of the total cost of the trip, $84 \mathrm{lei} /$ person was spent for the entrance fees related to the 13 sights of a total of 35 (Table 4).

Totalising the costs of all tourist services that were used during the trip, the final price was of $625 \mathrm{lei} / \mathrm{student}$ for the school trip, whereas for the academic trip the final price was 585 lei/undergraduate. Even though the difference between the two final costs was caused by the costs of the transportation services, they had the smallest proportion in the total, the highest costs being the catering ones, followed by the accommodation ones.

The trip planner contains the programme of the organised activities, taking into account the time resources allotted for the visitation of the tourist attractions, meal times, and the time allotted for the coach to go the distance between the objectives (Table 6). We have also considered the time that students needed to accomplish their tasks, but also to deepen their knowledge, through these generically called "visits".

\section{Analysis of students' and undergraduates' activities}

Both categories of tasks, for students and for undergraduates were meant for all the three stages of the trip starting with the preparation phase and ending with the evaluation one (Table 7).

For the students, the initial stage was less demanding, consequently after they got familiar to the extracurricular activity, the general data of the trip and after getting informed about the contents of the worksheets and of the portfolios, they immediately went to the actual research stage. Therefore, each student made a short informative summary on the tourist attraction they had drawn. Consulting the bibliographic materials and the online available information, the students started drawing the most interesting information on the set sight. They created presentations that were interesting for their colleagues, thus increasing their chances of receiving a prise in "The Junior Tour Guide: best presentation" Contest.

The undergraduates had a research stage with a higher degree of complexity, in which they started their research right after details of the trip were clear and the tasks shared. For each tourist sight, they had to identify its situation regarding its online visibility (whether it had its own site, a forum, a page on socialisation networks, or if it was mentioned on specialised tourist sites, etc.). The acquired information was written down in the corresponding fields of the worksheets, along with other observations and proposals. In pairs, the undergraduates, then made an essay containing the presentation of the sight assigned by the professor in a most unique and original manner, in order to impress their colleagues with curiosities and thus getting the highest score for the "Best Tour Guides" Contest.

The field stage, which also included solving the assigned tasks on the spot, referred to presenting the previously prepared presentations, thus 
having the students/undergraduates playing the part of the tour guide in turns. Each participant filled in a worksheet for each visited tourist attraction. The tasks were solved, according to preference, either on the spot, or on the coach, after the visit, or in their rooms, in the evening.

The tasks required a process of analysis and observation which was more simple in the case of the students (to identify, evaluate, classify and compare) and much more complex in the case of the undergraduates (specific matters of tourist management and development, valorisation of tourism potential, comparison of the observed images - the one on site and the online one, to identify the suitable forms of tourism, etc. ).

In Figure 4, there is an example of worksheet filled in by a student (fictitious name), and in Figure 5, there is one filled in by an undergraduate (fictitious name). Students included all materials in their portfolios and the undergraduates in their trip notebooks and these they handed in for evaluation at the end of the trip.

TOURISTIC ATTRACTION: Bârsana Monastery Locality: Bârsana

$\checkmark \quad$ Identifying strengths and weaknesses of the touristic attraction

\begin{tabular}{|c|c|}
\hline Strengths & Weaknesses \\
\hline $\begin{array}{ll}- & \text { unicity } \\
\text { - } & \text { architecture } \\
\text { natural environment }\end{array}$ & - $\quad$ lack of sanitary equipment \\
\hline
\end{tabular}

$\checkmark \quad$ Evaluation point score for the visited touristic attraction: $10 \mathrm{pt}$.

$\checkmark$ Evaluation point score for colleague's presentation: $10 \mathrm{pt}$.

$\checkmark \quad$ Classifying tourist attraction - situate within subcategory:

\begin{tabular}{|c|c|}
\hline Natural touristic elements & Anthropic touristic elements \\
\hline $\begin{array}{ll}> & \text { Relief } \\
> & \text { Climate } \\
> & \text { Water network } \\
> & \text { Flora and fauna }\end{array}$ & $\begin{array}{ll}\checkmark & \text { Archaeological and historical } \\
& \text { vestiges } \\
\checkmark & \text { Churches and monasteries } \\
\checkmark & \text { Cultural buildings and } \\
& \text { monuments } \\
\checkmark & \begin{array}{l}\text { Crafts and traditional } \\
\text { customs }\end{array}\end{array}$ \\
\hline
\end{tabular}

- subcategory: churches and monasteries

$\checkmark$ Comparative assessment of subcategories' components (touristic resort, monastery, water storage, castrum, museum, etc.)

Advantages of the analysed

\begin{tabular}{|c|c|}
\hline $\begin{array}{c}\text { Advantages of the analysed } \\
\text { attraction }\end{array}$ & Disadvantages of the analysed attraction \\
\hline$\bullet \quad$ museum & $\bullet \quad$ faraway from the main cities \\
$\bullet \quad$ landscape & souvenirs
\end{tabular}

Fig. 4. Students' worksheet 


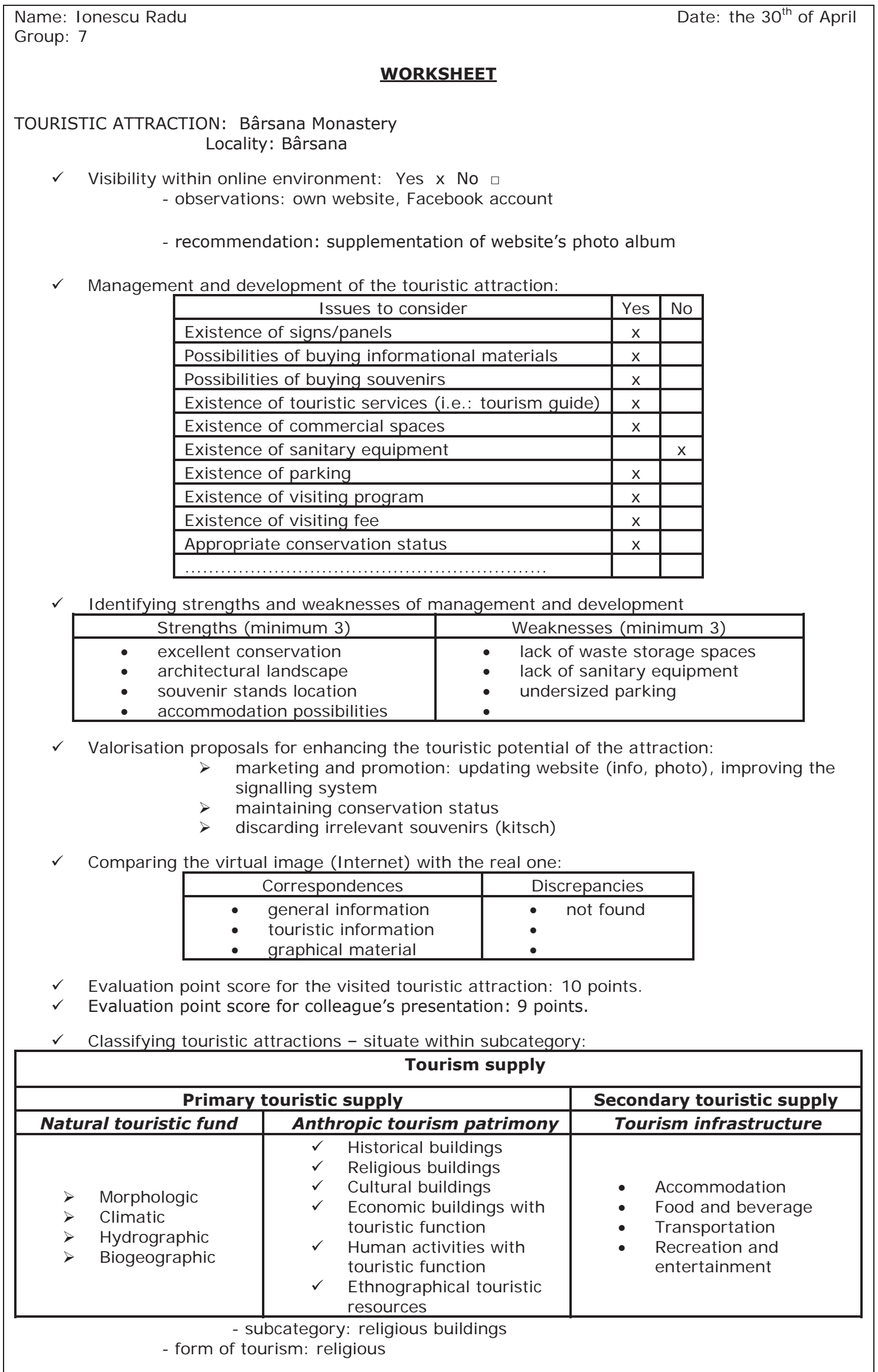




\begin{tabular}{|c|c|c|}
\hline \multirow[t]{3}{*}{$\checkmark$} & \multicolumn{2}{|c|}{$\begin{array}{l}\text { Comparative assessment of subcategories' components (touristic resort, monastery, } \\
\text { water storage, castrum, museum, etc.) }\end{array}$} \\
\hline & Advantages of the analysed attraction & $\begin{array}{l}\text { Disadvantages of the } \\
\text { analysed attraction }\end{array}$ \\
\hline & $\begin{array}{ll}\text { - } & \text { unicity } \\
\text { - } & \text { architectural style } \\
\text { - } & \text { natural environment }\end{array}$ & $\begin{array}{l}\text { - distant location toward the } \\
\text { - }\end{array}$ \\
\hline
\end{tabular}

Fig. 5. Undergraduates' worksheet

\section{Analysis of students and undergraduates results}

The students were evaluated according to their informative summaries previously prepared, to their presentation within the "Junior Tour Guidebest presentation" Contest, and according to their portfolios consisting of 35 worksheets filled in for each visited sight, and according to the minimum number of 7 suggestive photos.

The undergraduates' evaluation focused on three directions: the essay, the guiding moment for "The Best Tour Guides" Contest; the trip notebook consisting of 35 worksheets filled in for each tourist attraction that was visited, and the minimum of 12 photos related to the information to be found within the notebook.

Consequently, the best three tour guides were chosen from among the students, as well as the best three guiding pairs from among the undergraduates, but also the owners of the most valuable student portfolios and undergraduate notebooks, whose selection was based on information pertinence, originality of observations and relevance of photos.

\section{Similarities and differences between the school trip and the academic}

A series of similarities and differences can be seen between the school trip and the scientific educational one: the threefold structure of the organisational stages and the development (before, during and after the trip phases); the students' and undergraduates' involvement degree caused by the request to prepare the proper presentations for the visited tourist attractions and the filling in and handing in of some portfolios and trip notebooks (medium in the initial phase, high in the field stage, and low in the final stage); the evaluation forms (the presentation of the tourist sight and the worksheets); the general educational instructive purpose of the trip; the varied and complex thematic of the trip (morphologic and relief components, hydro-geographic, historical, cultural and religious buildings, elements of ethnography etc.).

The similarities also concern the organisational and tourist side: organisation form (under the coordination and guidance of the teachers and professors), region of the trip's unfolding (North-West), type of itinerary (tour), season (spring), duration of the trip (5 days), visited localities, form and contents of the trips' planner over the 5 days. There are similarities in 
what regards the tourist services as well: the chosen company and means of transportation (by coach), accommodation services (the same hotels and guest houses), catering services (the restaurants of the accommodation units or those in the vicinity of the tourist attractions), and the amenities (the same sights were visited by both students and undergraduates as well as).

There are, however, some differences between the school trip and the scientific educational trip, illustrated in Table 8 below.

Table 8. Differences between school trip and academic trip

\begin{tabular}{|c|c|c|}
\hline $\begin{array}{l}\text { Analysis } \\
\text { criterion }\end{array}$ & School trip & Academic trip \\
\hline Level of education & $\begin{array}{l}\text { Pre-university: secondary } \\
\text { school ( } 8^{\text {th }} \text { grade) }\end{array}$ & $\begin{array}{l}\text { Superior: Bachelor level } \\
\text { ( } 1^{\text {st }} \text { year of study) }\end{array}$ \\
\hline $\begin{array}{l}\text { Number of } \\
\text { participants }\end{array}$ & $\begin{array}{l}46 \text { students and } 4 \\
\text { teachers }\end{array}$ & 70 undergraduates and 5 professors \\
\hline Participants' age & 14-15 years old & $19-23$ years old \\
\hline Status of activities & optional & compulsory (credits) \\
\hline Number of tasks & $\begin{array}{l}8 \text { (of which } 5 \text { in the } \\
\text { worksheet) }\end{array}$ & 13 (of which 9 in the worksheet) \\
\hline $\begin{array}{l}\text { Complexity of } \\
\text { tasks }\end{array}$ & $\begin{array}{l}\text { - medium to easy } \\
\text { (general): to prepare the } \\
\text { summary, to make the } \\
\text { presentation, to observe, } \\
\text { to identify, to categorise, } \\
\text { to classify, to compare }\end{array}$ & $\begin{array}{l}\text { - medium to difficult (specific): to } \\
\text { prepare the essay, supplementary } \\
\text { research in the initial stage, to make the } \\
\text { presentation, to observe, to analyse, to } \\
\text { identify, to categorise, to classify, to } \\
\text { compare, to issue value judgements and } \\
\text { make proposals }\end{array}$ \\
\hline Evaluation product & Portfolio & Trip notebook \\
\hline Total costs & 625 lei/pers. & 585 lei/pers. \\
\hline
\end{tabular}

\section{CONCLUSIONS}

At the end of the research, it was concluded that organising and seeing through extracurricular activities both in the pre-university and within the academic environment required complete harmony between the administrative tourist side and the educational one, so that the students and the undergraduates would benefit completely from their partaking in such activities.

In the organisation of the two activities, there were similar stages: the one previous to the trip (initial), the field stage and the after the trip phase (final). In the first stage, the participants were informed about the tourist attractions, conditions, activities, tasks and research on the sights on route. In the field stage, students and undergraduates alike had to solve tasks that involved cognitive processes of identification, classification, and comparison, subordinate and in close relation with the tourist resources.

In the final stage, the students' and undergraduates' results were evaluated, the portfolios and the trip notebooks respectively. From a touristic perspective, the most important stage was the one before the trip, since it was 
then when the details about the trip planner and tourist services were set up (transportation, accommodation, catering, entertaining, etc.). From an educational point of view, the field stage was the most valuable.

Comparing the two forms of non-formal educational activities, the school trip and the scientific educational academic trip, we noticed similarities and differences among them on both the tourist-administrative level and on the educational one. Thus, we identified a series of similarities between the two trips, especially those related to the theme, purpose, evaluation form, participants' involvement degree, structure, and the involved tourist elements (from tourist resources to services). We also identified the differences: the educational level, the age and number of the participants, the quantitative and qualitative particularities of the tasks; the complexity of the evaluation product (students' portfolios and the undergraduates' notebooks).

\section{ACKNOWLEDGMENTS}

This work was possible due to the financial support of the Sectorial Operational Program for Human Resources Development 2007-2013, co-financed by the European Social Fund, under the project number POSDRU/159/1.5/S/132400 with the title "Young Successful Researchers - Professional Development in an International and Interdisciplinary Environment".

\section{References}

Blândul, V. C. (2008). Educaţia non-formală: de la teorie la practică. Oradea: Editura Universităţii din Oradea.

Bocoş, M., \& Jucan, D. (2008a). Fundamentele pedagogiei: teoria şi metodologia curricumului: repere şi instrumente didactice pentru formarea profesorilor. Piteşti: Editura Paralela 45.

Bocoş, M., \& Jucan, D. (2008b). Teoria şi metodologia instruirii şi teoria şi metodologia evaluării: repere şi instrumente didactice pentru formarea profesorilor. Ediţia a III-a. Piteşti: Editura Paralela 45.

Bradea, A. (2012). Educaţia nonformală în tabere: îndrumar de bune practici. Oradea: Editura Universităţii din Oradea.

Costea, O. (2009). Educaţia nonformală şi informală: realităţi şi perspective în şcoala românească. Bucureşti: Editura Didactică şi Pedagogică.

Cristea, S. (2006). Curriculum pedagogic: pentru formarea personalului didactic. Bucureşti: Editura Didactică şi Pedagogică.

Cucoş, C. (2006). Pedagogie. Iaşi: Editura Polirom. 
Frant, A. (2003). Excursia şcolară: proiectare, realizare, valorificare. Timişoara: Editura Solness.

Moşoarcă, I. I. (2005). Excursia geografică şi valenţele educative. Lugoj: Editura Nagard.

MEN. (2011). Legea Educaţiei Naţionale [Law on National Education]. Law no. 1/2011, published in Monitorul Oficial, Part I, no. 18, January 10 ${ }^{\text {th }}, 2011$.

http://distanta.ro/, Retrieved April 22, 2015

http://www.tinuta.ro/, Retrieved April 22, 2015

http://www.cursbnr.ro/, Retrieved April 22, 2015

http://www. plecat.ro/cazare/romania/cluj/ baisoara/hotel--1390--hotel-alpin-

baisoara.htm, Retrieved April 22, 2015

http://www. infopensiuni.ro/cazare-valea-draganului/\#, Retrieved April 22, 2015

http://www. pensiuneagiurgiuman.ro/tarife, Retrieved April 22, 2015

http://www. bicar.ro/cazare-valea-draganului-pensiune-valea-draganului.html, Retrieved April 22, 2015

http://www.turistinfo.ro/sighetu_marmatiei/cazare-sighetu_marmatiei/hotel_novac83177.html, Retrieved April 22, 2015

http://www.hotelbuti.ro/en/index.php?en_rooms-and-rates\%2C12, Retrieved April 22, 2015

http://www. hotel-gabriela.ro/ro/servicii-cazare/oferta-si-tarife-cazare, Retrieved April 22, 2015

http://www.cabanamotilor.ro/servicii.html, Retrieved April 22, 2015

http://salinaturda.eu/, Retrieved April 22, 2015

http://www.muzeuloctaviangoga.ro/, Retrieved April 22, 2015

http://www.gradina-botanica-jibou.ro/, Retrieved April 22, 2015

http://www. muzeuminbm.ro/, Retrieved April 22, 2015

http://www.memorialsighet.ro/, Retrieved April 22, 2015

http://muzeulmaramuresului.ro/, Retrieved April 22, 2015

http://www.manastireabarsana.ro/, Retrieved April 22, 2015

http://www.cffviseu.ro/content/ro/mersul-trenurilor-cu-abur, Retrieved April 22, 2015

http://complexulmuzealbn.ro/sectii/muzeul-george-cosbuc-cosbuc, Retrieved April 22, 2015 